\title{
Low-frequency scalar absorption cross sections for stationary black holes
}

\author{
Atsushi Higuchi \\ Department of Mathematics, University of York \\ Heslington, York, YO10 5DD, UK \\ Email: ah28@york.ac.uk
}

1 November, 2001

\begin{abstract}
We discuss the absorption cross section for the minimally-coupled massless scalar field into a stationary and circularly symmetric black hole with nonzero angular velocity in four or higher dimensions. In particular, we show that it equals the horizon area in the zero-frequency limit provided that the solution of the scalar field equation with an incident monochromatic plane wave converges pointwise to a smooth time-independent solution outside the black hole and on the future horizon, with the error term being at most linear in the frequency. We also show that this equality holds for static black holes which are not necessarily spherically symmetric. The zero-frequency scattering cross section is found to vanish in both cases. It is shown in an Addendum that the equality holds for any stationary black hole with vanishing expansion if the limit solution is known to be a constant.
\end{abstract}

Properties of black holes have been attracting much attention in connection with string theory since it was found that the Bekenstein-Hawking entropy is reproduced by string theory for some black holes [ []]. The absorption cross section for the minimallycoupled massless scalar field was studied in this context [2, 3, 国, 5], and Das, Gibbons and Mathur showed that it is exactly the horizon area for a static and spherically symmetric black hole in any dimensions in the zero-frequency limit [6]. (See, e.g. [0, 8, 9, 10] for earlier calculations of absorption cross sections for black holes.) It is natural to 
ask whether the same result holds for rotating black holes. In fact Maldacena and Strominger have shown [11], among other things, that this equality is valid for the four-dimensional Kerr-Newman black hole. This result has been generalized, and the equality has been verified for other rotating black holes as well [12, 13, 14]. However, there has been no general explanation of this universality so far except for the original work dealing with the static and spherically symmetric case. In this Letter we show that this equality holds for any stationary and circularly symmetric black hole with nonzero angular velocity in any spacetime dimensions larger than three if the solution of the scalar field equation with an incident monochromatic plane wave converges pointwise to a smooth time-independent solution in the zero-frequency limit outside the black hole and on the future horizon, with the error term being at most linear in the frequency. [We call the solution with an incident monochromatic plane wave the asymptotically-plane-wave (APW) solution.] We also show the same equality for any static black hole, which may not be spherically symmetric. Then we show that the zero-frequency scattering cross section vanishes for these black holes.

By definition a static black-hole spacetime admits a Killing vector field (generating time translations) which is hypersurface-orthogonal, timelike outside the black hole (or in the domain of outer communications [15]) and null on the future and past horizons. Thus, the metric can be written outside the black hole as

$$
\left.d s^{2}\right|_{\text {static }}=-N^{2} d t^{2}+h_{m n}^{(s)} d x^{m} d x^{n}
$$

where $N^{2}(>0)$ and $h_{m n}^{(s)}$ (which is a positive-definite matrix) are independent of $t$. In $(p+2)$-dimensional stationary and circularly symmetric black-hole spacetime, the 2surfaces generated by the commuting Killing vectors $(\partial / \partial t)^{a}$ and $(\partial / \partial \phi)^{a}$ are orthogonal to another family of $p$-dimensional surfaces by definition [15, 16]. (We assume that the latter surfaces are spacelike outside the black hole and on the horizons.) Moreover, the spacetime is periodic in $\phi$ with period $2 \pi$. Thus, the metric can be made $t$ - and $\phi$-independent and written outside the black hole as

$$
\left.d s^{2}\right|_{\text {circular }}=-N^{2} d t^{2}+h_{\phi \phi}\left(d \phi-N^{\phi} d t\right)^{2}+h_{m n}^{(c)} d x^{m} d x^{n},
$$

where the function $h_{\phi \phi}$ and the matrix $h_{m n}^{(c)}$ are positive definite.

In both cases, we assume that compact spacelike p-surfaces of constant $t$ foliate the future horizon' and that the spacetime is asymptotically flat. In the circularly symmetric case, we assume that the null generator of the future horizon is of the form $(\partial / \partial t)^{a}+$

\footnotetext{
${ }^{1}$ The variable $t$ here differs from that in (11) or (2) by addition of a function of the other variables.
} 
$\Omega_{H}(\partial / \partial \phi)^{a}$ with the constant $\Omega_{H}$ being nonzero, and that the p-surfaces foliating the future horizon are invariant under $\phi$-rotations.] [A stationary black hole in general relativity in four dimensions is either static or circularly symmetric and satisfies the conditions required here under reasonable assumptions (see [17, 15]).]

Let us first derive a useful formula for the absorption cross section for any stationary black hole. Far away from the black hole, the APW solution, $\psi=\Psi_{\omega} e^{-i \omega t}, \omega>0$, of the equation $\nabla_{a} \nabla^{a} \psi=0$ in $p+2$ dimensions is approximately given by

$$
\Psi_{\omega} \approx e^{i \omega r \cos \theta}+f_{\omega}(\Omega) \frac{e^{i \omega r}}{r^{p / 2}} .
$$

Here, $\Omega$ represents all angular variables on the unit $p$-sphere, and $r$ is the approximate distance from the black hole. The variable $\theta$ is the angle between the position vector and the direction in which the incident wave propagates. There is a conserved current given by

$$
J_{a}=-i\left(\psi^{*} \nabla_{a} \psi-\psi \nabla_{a} \psi^{*}\right),
$$

where the star denotes complex conjugation. The current flowing out of the past horizon must be zero for the APW solution. This requirement will be important later. For the incident wave $e^{i \omega r \cos \theta}$ in (31) we have $n^{a} J_{a}=-J_{t}=2 \omega$ with $n^{a}$ being the unit vector in the direction of propagation. Therefore, the absorption cross section is $(2 \omega)^{-1}$ times the rate of this current going across the future horizon integrated over the horizon area.

A stationary black hole has the future and past horizons which are null and fixed under time translations. The spacetime metric in a neighbourhood of the future horizon, parametrized by $x^{i}(i=1, \ldots, p), \lambda$ and $t$, can be chosen as

$$
d s^{2}=-A d t^{2}+g_{i j}\left(d x^{i}-C^{i} d t\right)\left(d x^{j}-C^{j} d t\right)+2 B d \lambda d t,
$$

where $A=0$ at $\lambda=0$ (on the horizon) and $A>0$ for $\lambda>0$ (outside the horizon). The quantities $B(>0), A, C^{i}$ and the positive-definite matrix $g_{i j}$ are $t$-independent. This metric can be obtained as follows. Let the line element of the compact spacelike $p$-surfaces, $S_{t}$, of constant $t$, which foliate the future horizon, be $g_{i j} d x^{i} d x^{j}$. Then the line element on the horizon is $g_{i j}\left(d x^{i}-C^{i} d t\right)\left(d x^{j}-C^{j} d t\right)$ for some $C^{i}$. Consider the past-directed and outward-pointing null geodesics which are orthogonal to $S_{t}$. Let $t$ and $x^{i}$ be constant on each of these geodesics and let $\lambda$ be its affine parameter. Then the metric is of the form (5) since these geodesics are orthogonal to $\left(\partial / \partial x^{i}\right)^{a}$ for all $\lambda$.

A $(p+1)$-dimensional hypersurface of constant $\lambda$ is timelike for $\lambda>0$ and null for $\lambda=0$. If we write the unit normal to the constant $\lambda$ hypersurface with $\lambda>0$ which

\footnotetext{
${ }^{2}$ We will not explicitly use the corresponding statements for the past horizon.
} 
points towards the horizon as $-A^{-1 / 2} l^{a}$, then

$$
l^{a}=(\partial / \partial t)^{a}+B^{-1} A(\partial / \partial \lambda)^{a}+C^{i}\left(\partial / \partial x^{i}\right)^{a} .
$$

Note that the vector $l^{a}$ on the horizon,

$$
\left.l^{a}\right|_{\text {horizon }}=(\partial / \partial t)^{a}+C^{i}\left(\partial / \partial x^{i}\right)^{a},
$$

is the null generator of the horizon. The volume element of the hypersurface of constant $\lambda$ is $A^{1 / 2} \sqrt{g}$. Therefore, the absorption cross section is

$$
\begin{aligned}
\sigma_{\omega} & =-\frac{1}{2 \omega} \int d^{p} x \sqrt{g} l^{a} J_{a} \\
& =\int d^{p} x \sqrt{g}\left|\Psi_{\omega}\right|^{2}-\frac{1}{\omega} \operatorname{Im} \int d^{p} x \sqrt{g} C^{i} \Psi_{\omega}^{*} \partial_{i} \Psi_{\omega},
\end{aligned}
$$

where integration is performed on the future horizon. If

$$
\lim _{\omega \rightarrow 0}\left|\Psi_{\omega}\right|^{2}=1
$$

and

$$
\lim _{\omega \rightarrow 0} \frac{1}{\omega} \int d^{p} x \sqrt{g} C^{i} \Psi_{\omega}^{*} \partial_{i} \Psi_{\omega}=0
$$

on the future horizon, then it follows from (8) that the zero-frequency absorption cross section $\sigma_{0}$ equals the horizon area $\int d^{p} x \sqrt{g}$. We will show that equations (9) and (10) indeed hold under the assumptions stated before. We will first assume in addition that

$$
\Psi_{0} \equiv \lim _{\omega \rightarrow 0} \Psi_{\omega} \approx 1+\mathcal{O}\left(r^{-p / 2}\right)
$$

for large $r$, where $\mathcal{O}\left(r^{-\beta}\right)$ denotes a term of order $r^{-\beta}$ or smaller. This assumption will be justified later for $p \geq 2$."]

For the static case we have $C^{i}=0$ on the horizon because the Killing vector $(\partial / \partial t)^{a}$ is orthogonal to the horizon. Thus, equation (10) is trivially satisfied. Now, $f_{0} \equiv \Psi_{0}-1$ is a time-independent solution of $\nabla_{a} \nabla^{a} f_{0}=0$ falling off at least as fast as $r^{-p / 2}$ for large $r$ due to (11). (We assume that $f_{0}$ is real. If this is not the case, one can consider the real and imaginary parts of $f_{0}$ separately.) Consider the spacetime integral of $\nabla_{a} f_{0} \nabla^{a} f_{0}$ in the region outside the horizon between two spacelike hypersurfaces which intersect the future horizon and are orthogonal to $(\partial / \partial t)^{a}$ far away from the black hole. Let one of the hypersurfaces be obtained by a time translation of the other. Then we have

$$
\begin{aligned}
\int d V \nabla_{a} f_{0} \nabla^{a} f_{0} & =\int d V \nabla_{a}\left(f_{0} \nabla^{a} f_{0}\right) \\
& =-\int_{\text {horizon }} d t d^{p} x \sqrt{g} f_{0} \frac{\partial f_{0}}{\partial t}+\lim _{r \rightarrow \infty} \int d t d \Omega r^{p} f_{0} \frac{\partial f_{0}}{\partial r},
\end{aligned}
$$

${ }^{3}$ We have been unable to establish (11) for $p=1$, and this is why our result is limited to four or higher dimensions. 
where $d V$ is the spacetime volume element. The first term on the right-hand side vanishes because $f_{0}$ is time-independent. The second term is zero because of the fall-off condition (11). However, $\nabla_{a} f_{0} \nabla^{a} f_{0}$ is non-negative outside the black hole because

$$
\nabla_{a} f_{0} \nabla^{a} f_{0}=h^{(s) m n} \partial_{m} f_{0} \partial_{n} f_{0}
$$

where $h^{(s) m n}$ is the inverse of $h_{m n}^{(s)}$ in (1). Thus, equation (12) implies that $\nabla_{a} f_{0}=0$. Since $f_{0} \rightarrow 0$ as $r \rightarrow \infty$, we must have $f_{0}=0$, i.e. $\Psi_{0}=1$ outside the black hole. (This is a special case of a no-hair theorem due to Bekenstein [18].) Hence, equation (9) holds on the future horizon by the assumed smoothness of the solution.

Next let us discuss the case with a stationary and circularly symmetric black hole. Here, we choose $\phi$ as one of the $x^{i}$ s in (5). Then the metric $g_{i j}$ is $\phi$-invariant by assumption. As we stated before, we assume that the null generator (7) of the future horizon is $(\partial / \partial t)^{a}+\Omega_{H}(\partial / \partial \phi)^{a}$, where $\Omega_{H}$ is a nonzero constant interpreted as the angular velocity of the horizon. Thus, we have on the horizon

$$
C^{i}\left(\partial / \partial x^{i}\right)^{a}=\Omega_{H}(\partial / \partial \phi)^{a}
$$

Let us write

$$
\Psi_{0} \equiv \sum_{m=-\infty}^{+\infty} \Psi_{0}^{(m)},
$$

where $\partial_{\phi} \Psi_{0}^{(m)}=i m \Psi_{0}^{(m)}$ with $\partial_{\phi}=\partial / \partial \phi$. The (suitably normalized) component of the current going into the future horizon for the solution $\Psi_{0}^{(m)}$ is $-l^{a} J_{a}=-2 m \Omega_{H}\left|\Psi_{0}^{(m)}\right|^{2}$. By time independence of the solution we conclude that this equals the current coming out of the past horizon. Thus, for $m \neq 0$, the part proportional to $e^{i m \phi}$ of the current coming out of the past horizon would be nonzero if $\Psi_{0}^{(m)} \neq 0$ on the future horizon. Since this could not be the case for (the $\omega \rightarrow 0$ limit of) the APW solution, we conclude that $\Psi_{0}^{(m)}=0$ for all nonzero $m$. Therefore, we have $\partial_{\phi} \Psi_{0}=0$ and $\Psi_{0}=\Psi_{0}^{(0)}$ on the future horizon. Then, $\partial_{\phi} \Psi_{\omega}=\mathcal{O}(\omega)$ for small $\omega$ by the assumption that the error term is at most linear in $\omega$. Thus, equation (10) is satisfied because

$$
\int d^{p} x \sqrt{g} C^{i} \Psi_{\omega}^{*} \partial_{i} \Psi_{\omega}=\Omega_{H} \int d^{p} x \sqrt{g} \Psi_{\omega}^{*} \partial_{\phi} \Psi_{\omega}=\mathcal{O}\left(\omega^{2}\right)
$$

due to (13). Now, outside the black hole we find from (2)

$$
\nabla_{a} f_{0} \nabla^{a} f_{0}=h^{(c) m n} \partial_{m} f_{0} \partial_{n} f_{0}+\left[h^{\phi \phi}-\left(N^{\phi}\right)^{2} / N^{2}\right]\left(\partial_{\phi} f_{0}\right)^{2}
$$

where $h^{(c) m n}$ is the inverse of $h_{m n}^{(c)}$ in (2) and $h^{\phi \phi} \equiv\left(h_{\phi \phi}\right)^{-1}$. Therefore, $\nabla_{a} f_{0} \nabla^{a} f_{0}$ is non-negative because $\partial_{\phi} f_{0}=0$. Hence, we must have $f_{0}=0$ and $\Psi_{0}=1$ outside the 
black hole because essentially the same argument as in the static case is valid [19. Thus, equation (9) holds on the future horizon again by the assumed smoothness.

Next, let us show equation (11), which enabled us to use the no-hair theorems, for $p \geq 2$. Far away from the horizon, the solution can be expanded as

$$
\Psi_{\omega} \approx \sum_{l=0}^{\infty} R_{l \omega}(r) Y_{l}(\Omega)
$$

where $Y_{l}(\Omega)$ is one of the spherical harmonics satisfying $\tilde{\Delta} Y_{l}(\Omega)=-l(l+p-1) Y_{l}(\Omega)$. Here, $\tilde{\Delta}$ is the Laplacian on the unit $p$-sphere. The radial function $R_{l \omega}(r)$ can be written as

$$
\begin{aligned}
R_{l \omega}(r) & \approx c_{l p}(\omega r)^{-(p-1) / 2}\left[\left(1+\alpha_{l \omega}\right) J_{l+(p-1) / 2}(\omega r)-i\left(1-\alpha_{l \omega}\right) N_{l+(p-1) / 2}(\omega r)\right] \\
& =c_{l p}(\omega r)^{-(p-1) / 2}\left[H_{l+(p-1) / 2}^{(1) *}(\omega r)+\alpha_{l \omega} H_{l+(p-1) / 2}^{(1)}(\omega r)\right] .
\end{aligned}
$$

Here, the functions $J_{\nu}(x)$ and $N_{\nu}(x)$ are the Bessel and Neumann functions, respectively, and $H_{\nu}^{(1)}(x)=J_{\nu}(x)+i N_{\nu}(x)$ is the Hankel function of the first kind. (See, e.g. 20] for the definitions of these functions.) The constants $c_{l p}$, which depend only on $l$ and $p$, are determined from the expansion of the plane wave $e^{i \omega r \cos \theta}$, and the first term in the second line of (16) is independent of the details of spacetime because it represents the wave coming in from $r=\infty$.

Now, the function $N_{\nu}(x)$ behaves like $x^{-\nu}$ whereas the function $J_{\nu}(x)$ behaves like $x^{\nu}$ for small $x$ if $\nu>0$. Therefore, for $R_{l \omega}(r)$ given by (16) to have a finite limit as $\omega \rightarrow 0$ for large $r$, we must have $1-\alpha_{l \omega}=\mathcal{O}\left(\omega^{l+p-1}\right)$. Then, we find

$$
\lim _{\omega \rightarrow 0} R_{l \omega}(r) \approx \mathcal{O}\left(r^{-l-p+1}\right)
$$

for large $r$ if $l>0$. To find the behaviour of the $s$-wave radial function, $R_{0 \omega}(r)$, we note first that $\sum_{l=0}^{\infty} R_{l \omega}(r) Y_{l}(\Omega)=e^{i \omega r \cos \theta}$ if $\alpha_{l \omega}=1$ for all $l$ and $\omega$. This implies that

$$
\lim _{r \rightarrow 0} 2 c_{0 p}(\omega r)^{-(p-1) / 2} J_{(p-1) / 2}(\omega r) Y_{0}(\Omega)=1 .
$$

Since the $r \rightarrow 0$ and $\omega \rightarrow 0$ limits are equivalent on the left-hand side of this equation and since $\alpha_{0 \omega} \rightarrow 1$ as $\omega \rightarrow 0$, we find

$$
\lim _{\omega \rightarrow 0} R_{0 \omega}(r) Y_{0}(\Omega) \approx 1+\mathcal{O}\left(r^{-(p-1)}\right)
$$

for large $r$. We deduce equation (11) from (17) and (19) because $l+p-1 \geq p / 2$ if $p \geq 2$.

Finally, let us show that the scattering cross section vanishes. The contribution of the $l$-th partial wave to the scattering cross section is at most of order $\omega^{2 l+p-2}$ for small 
$\omega$ because it is a constant multiple of $\omega^{-p}\left|1-\alpha_{l \omega}\right|^{2}$. [The contribution to the absorption cross section is $\omega^{-p}\left(1-\left|\alpha_{l \omega}\right|^{2}\right)$ times a numerical constant.] Hence, it vanishes in the zero-frequency limit unless $p=2$ and $l=0$. For $p=2$, i.e. in four dimensions, we find $\Psi_{0} \approx 1+K / r$ with the scattering cross section being $4 \pi|K|^{2}$. Since $K=0$, the $s$-wave contribution to the scattering cross section must also vanish.

\section{Addendum}

In fact, if the APW solution is known to converge to one (after a suitable adjustment of the phase), then the equality of the zero-frequency absorption cross section and the horizon area holds for any stationary black hole with vanishing expansion. (It is well known that stationary black holes must have horizons with vanishing expansion in general relativity if appropriate energy conditions are satisfied [17, 15].)

Let us first show that the horizon area is well defined if and only if $D_{i} C^{i}=0$ on the horizon, where $D_{i}$ is the convariant derivative compatible with the metric $g_{i j}$. Note that the metric (5) will still be $t$-independent after an (infinitesimal) transformation $t \rightarrow t+\epsilon$, where $\epsilon$ is $x^{i}$-dependent but $t$-independent. The metric $g_{i j}$ changes as $g_{i j} \rightarrow$ $g_{i j}-C_{i} D_{j} \epsilon-C_{j} D_{i} \epsilon$ under this transformation. Then the horizon area changes as

$$
\int d^{p} x \sqrt{g} \rightarrow \int d^{p} x \sqrt{g}\left(1+\epsilon D_{i} C^{i}\right)
$$

Hence, the horizon area is well defined if and only if $D_{i} C^{i}=0$. One can show that the expansion of the horizon generators is proportional to $D_{i} C^{i}$. Therefore, the horizon area is well defined if and only if the expansion of the horizon generators vanishes. Now, if $D_{i} C^{i}=0$, then equation (10) holds because

$$
\begin{aligned}
\int d^{p} x \sqrt{g} C^{i} \Psi_{\omega}^{*} D_{i} \Psi_{\omega} & =\int d^{p} x \sqrt{g} C^{i} \Psi_{\omega}^{*} D_{i}\left(\Psi_{\omega}-1\right) \\
& =-\int d^{p} x \sqrt{g} C^{i}\left(\Psi_{\omega}-1\right) D_{i} \Psi_{\omega}^{*}=\mathcal{O}\left(\omega^{2}\right) .
\end{aligned}
$$

Hence,

$$
\lim _{\omega \rightarrow 0} \sigma_{\omega}=\int d^{p} x \sqrt{g}\left|\Psi_{\omega}\right|^{2}=\int d^{p} x \sqrt{g}
$$

\section{Acknowledgements}

The author is grateful to John Friedman whose suggestions were essential in initiating this work. He also thanks Chris Fewster, Gary Gibbons and Bernard Kay for useful discussions. 


\section{References}

[1] A. Strominger and C. Vafa, Phys. Lett. B379, 99 (1996).

[2] S. R. Das and S. D. Mathur, Nucl. Phys. B478, 561 (1996).

[3] S. R. Das and S. D. Mathur, Nucl. Phys. B482, 153 (1996).

[4] S. S. Gubser and I. Klebanov, Nucl. Phys. B482, 173 (1996).

[5] J. Maldacena and A. Strominger, Phys. Rev. D 55, 861 (1997).

[6] S. R. Das, G. Gibbons and S. D. Mathur, Phys. Rev. Letters 78, 417 (1997).

[7] A. A. Starobinskii and S. M. Churilov, Sov. Phys. JETP 38, 1 (1974).

[8] G. W. Gibbons, Commun. Math. Phys. 44, 245 (1975).

[9] D. N. Page, Phys. Rev. D 13, 198 (1976).

[10] W. G. Unruh, Phys. Rev. D 14, 3251 (1976).

[11] J. Maldacena and A. Strominger, Phys. Rev. D 56, 4975 (1997).

[12] M. Cvetič and F. Larsen, Phys. Rev. D 56, 4994 (1997).

[13] M. Cvetič and F. Larsen, Nucl. Phys. B506, 107 (1997).

[14] G. W. Gibbons and C. A. R. Herdeiro, Class. Quantum Grav. 16, 3619 (1999).

[15] B. Carter, in General Relativity: An Einstein Centenary Survey, S. W. Hawking and W. Israel eds. (Cambridge University Press, Cambridge, 1979).

[16] B. Carter, Commun. Math. Phys., 30, 261 (1973).

[17] S. W. Hawking and G. F. R. Ellis, The Large Scale Structure of Space-Time, (Cambridge University Press, Cambridge, 1973).

[18] J. Bekenstein, Phys. Rev. D 5, 1239 (1972).

[19] J. Bekenstein, Phys. Rev. D 5, 2403 (1972).

[20] I. S. Gradshteyn and I. M. Ryzhik, Talbe of Integrals, Series, and Products, Allan Jeffrey ed. (Academic Press, New York, 1980). 